\title{
Genetic variations in Bestrophin-1 and associated clinical findings in two Chinese patients with juvenile-onset and adult-onset best vitelliform macular dystrophy
}

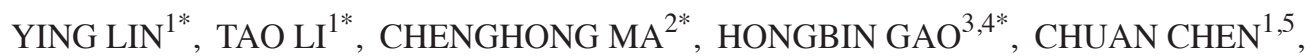 \\ YI ZHU ${ }^{1,5}$, BINGQIAN LIU ${ }^{1}$, YU LIAN ${ }^{1}$, YING HUANG ${ }^{1}$, HAICHUN LI ${ }^{1}$, QINGXIU WU ${ }^{1}$, \\ XIAOLING LIANG ${ }^{1}$, CHENJIN JIN $^{1}$, XINHUA HUANG ${ }^{1}$, JIANHUA YE ${ }^{2}$ and LIN LU ${ }^{1}$
}

\begin{abstract}
${ }^{1}$ State Key Laboratory of Ophthalmology, Zhongshan Ophthalmic Center, Sun Yat-sen University, Guangzhou, Guangdong 510060; ${ }^{2}$ Department of Endocrine, College of Clinical Medicine, The First Affiliated Hospital of Guangdong Pharmaceutical University, Guangzhou, Guangdong 510080; ${ }^{3}$ Guangdong Laboratory Animals Monitoring Institute, Key Laboratory of Guangdong Laboratory Animals, Guangzhou, Guangdong 510640;

${ }^{4}$ Department of Toxicology, School of Public Health and Tropical Medicine, Southern Medical University,

Guangzhou, Guangdong 510515, P.R. China; ${ }^{5}$ Department of Molecular and Cellular Pharmacology,

University of Miami Miller School of Medicine, Miami, FL 33136, USA
\end{abstract}

Received June 20, 2017; Accepted September 19, 2017

DOI: $10.3892 / \mathrm{mmr} .2017 .7927$

\begin{abstract}
Best vitelliform macular dystrophy (BVMD) is a hereditary retinal disease characterized by the bilateral accumulation of large egg yolk-like lesions in the sub-retinal and sub-retinal pigment epithelium spaces. Macular degeneration in BVMD can begin in childhood or adulthood. The variation in the age of onset is not clearly understood. The present study characterized the clinical characteristics of two Chinese patients with either juvenile-onset BVMD or adult-onset BVMD and investigated the underlying genetic variations. A 16-year-old male (Patient 1) was diagnosed with juvenile-onset BVMD and a 43-year-old female (Patient 2) was diagnosed with adult-onset BVMD. Comprehensive ophthalmic examinations were performed, including best-corrected visual acuity, intraocular pressure, slit-lamp examination, fundus photography, optical coherence tomography, fundus fluorescein angiography
\end{abstract}

Correspondence to: Dr Jianhua Ye, Department of Endocrine, College of Clinical Medicine, The First Affiliated Hospital of Guangdong Pharmaceutical University, 19 Nonglinxia Road, Guangzhou, Guangdong 510080, P.R. China

E-mail: jhyee2016@sina.com

Dr Lin Lu, State Key Laboratory of Ophthalmology, Zhongshan Ophthalmic Center, Sun Yat-sen University, 54 South Xianlie Road, Guangzhou, Guangdong 510060, P.R. China

E-mail:drlulinocular@126.com

${ }^{*}$ Contributed equally

Key words: best vitelliform macular dystrophy, bestrophin-1, juvenile-onset, adult-onset, mutation imaging and Espion electrophysiology. Genomic DNA was extracted from peripheral blood leukocytes collected from these patients, their family members, and 200 unrelated subjects within in the same population. The 11 exons of the bestrophin-1 (BEST1) gene were amplified by polymerase chain reaction and directly sequenced. Both patients presented lesions in the macular area. In Patient 1, a heterozygous mutation c.903T $>\mathrm{G}$ (p.D301E) in exon 8 of the BEST1 gene was identified. This mutation was not present in any of the unaffected family members or the normal controls. Polymorphism phenotyping and the sorting intolerant from tolerant algorithm predicted that the amino acid substitution D301E in bestrophin-1 protein was damaging. In Patient 2, a single nucleotide polymorphism c.1608C >T (p.T536T) in exon 10 of the BEST1 gene was identified. These findings expand the spectrum of BEST1 genetic variation and will be valuable for genetic counseling and the development of therapeutic interventions for patients with BVMD.

\section{Introduction}

Best vitelliform macular dystrophy (BVMD), also known as Best disease, is a hereditary retinal disease characterized by the bilateral accumulation of large egg yolk-like lesions in the sub-retinal and sub-retinal pigment epithelium (RPE) spaces $(1,2)$. This lesion may eventually break up and spread throughout the macular area, leading to central vision reduction and retinal detachment (3). The clinical manifestation of BVMD varies substantially in different disease stages and among individual patients (4). For example, retinal lesions in BVMD typically occur bilaterally and symmetrically; however, rarely, patients can exhibit unilateral maculopathy $(5,6)$. Patients with atypical BVMD presentation can have multifocal macular and extramacular involvement, including 
retinitis pigmentosa, microcornea, retinal dystrophy, cataract, and posterior staphyloma syndrome (6-13). Macular degeneration in BVMD can begin from childhood or adulthood, and is classified as juvenile-onset BVMD or adult-onset BVMD, respectively (14). The variation in the age of onset is not clearly understood, and few studies have compared the differences between these two classifications $(13,15)$. Currently, there is no effective treatment for BVMD.

BVMD is linked to the $15 \mathrm{~kb}$ bestrophin-1 (BEST1) gene, located on chromosome 11q12-q13. The BEST1 gene contains 11 exons, of which 10 are protein-coding (1). More than 250 disease-causing mutations in BEST1 have been reported to be associated with BVMD (16,17). The $68 \mathrm{kDa}$ bestrophin-1 protein, encoded by BEST1, is localized to the basolateral plasma membrane of RPE cells and contains several domains with a high degree of evolutionary conservation (18). The function of bestrophin-1 remains unclear. It is proposed to act as a chloride channel, activated by intracellular $\mathrm{Ca}^{2+}$ and/or as a channel regulator (19). It may regulate both the transepithelial electrical properties and the $\mathrm{Ca}^{2+}$ signaling of RPE (20). RPE cells with mutant BEST1 are unable to effectively metabolize photoreceptor outer segments, leading to the anomalous accumulation of lipofuscin (4). Bestrophin-1 also promotes epithelial-to-mesenchymal transition of renal collecting duct cells under the regulation of transforming growth factor $\beta 1$, which has a critical role during cell growth and differentiation (21-26). Furthermore, under pathological conditions such as neuroinflammation and neurodegeneration, bestrophin-1 can redistribute in reactive astrocytes from perisynaptic microdomains to the soma, promoting the release of $\gamma$-aminobutyric acid (27). Identification of the genetic variations in BVMD may improve the understanding of the etiology and pathogenesis of the disease, and provide cues for the development of potential therapeutic interventions.

This study aimed to characterize the clinical manifestations and investigate the underlying genetic variations of a 16-year-old male and 43-year-old female with juvenile-onset and adult-onset BVMD, respectively.

\section{Patients and methods}

Study subjects and clinical ophthalmic examinations. A 16-year-old male with juvenile-onset BVMD (Patient 1) and a 43-year-old female with adult-onset BVMD (Patient 2), both from southern China, were diagnosed at Zhongshan Ophthalmic Center (Guangzhou, P.R. China). Visual acuity was examined using the Early Treatment Diabetic Retinopathy Study chart (Precision Vision, La Salle, IL, USA) (28). Images of the anterior segment were captured using a BX 900 Slit Lamp (Haag-Streit, Bern, Switzerland). Measurements of the anterior segment were recorded with Pentacam HR version 70700 (Oculus VR, LLC, Wetzlar, Germany). Optical Coherence Tomography (OCT) was performed by Cirrus HD-OCT (Carl Zeiss Meditec, Inc., Dublin, CA, USA). Fundus photography and fundus fluorescein angiography (FFA) imaging was performed using a Heidelberg Retina Angiograph (Heidelberg Engineering, Heidelberg, Germany). Multifocal electroretinography (mfERG) was performed to assess the amplitudes of the rod and cone responses using the Espion electrophysiology system (Diagnosys LLC, Littleton,
MA, USA). Physical examinations were performed to exclude systemic diseases.

Sample collection and mutation screening. Venous blood samples were collected from the study subjects, their family members and 200 subjects without BVMD within the same population (15-48 years old; sex ratio: Male/female=108/92). Genomic DNA was extracted from peripheral blood leukocytes using standard protocols. Briefly, a total amount of $1 \mathrm{ml}$ of blood sample was collected from each subject, lysed by red blood cell lysis buffer (Sigma Aldrich, Merck KGaA, Darmstadt, Germany), and centrifuged at 2,000 x g for $5 \mathrm{~min}$ at room temperature. Genomic DNA was extracted from peripheral blood leucocytes using a DNA extraction kit (Qiagen $\mathrm{GmbH}$, Hilden, Germany) (29,30). Exons of the BEST1 gene were amplified by polymerase chain reaction (PCR) with primers as previously described (31-34). The primer sequences are listed in Table I. PCR was conducted in a $50 \mu 1$ reaction system. using the PCR amplification kit (Takara Bio, Inc., Otsu, Japan). The amplification included a single 5 min step at $94^{\circ} \mathrm{C}$, followed by 40 cycles of $94^{\circ} \mathrm{C}$ for $45 \mathrm{sec}, 58-61^{\circ} \mathrm{C}$ for $45 \mathrm{sec}$, $72^{\circ} \mathrm{C}$ for $45 \mathrm{sec}$ and a final $10 \mathrm{~min}$ step at $72^{\circ} \mathrm{C}$. The PCR products were sequenced from both directions with an ABI3730 Automated Sequencer (PE Biosystems Inc., Foster City, CA). Sequenced products were analyzed using Seqman (version 2.3; Technelysium Pty Ltd., Brisbane, Australia), and compared with reference sequences in the database at the National Center for Biotechnology Information (NC_000011.10).

To analyze the effect of missense variants, polymorphism phenotyping (PolyPhen)and the sorting intolerant from tolerant (SIFT) algorithms were used to predict the possible impact of an amino acid substitution on the protein structure and function, using straightforward physical and comparative considerations (35-39). Variants were considered to be pathogenic when at least one of the two programs predicted a deleterious effect of the amino acid substitution on the protein structure and function. The Human Gene Mutation Database (http://www.hgmd.cf.ac.uk/ac/index.php) was used to screen mutations reported in published studies. HomoloGene (https:/www.ncbi.nlm.nih.gov/homologene) was used to check whether the mutated amino acid residues were conserved across different species.

Ethics. All experimental protocols and the methods were carried out in accordance with the guidelines approved by the ethics committee of Zhongshan Ophthalmic Center of Sun Yat-sen University (Guangzhou, P.R. China). Written informed consent was obtained from each subject in accordance with The Declaration of Helsinki. All participants provided informed consent for the publication of their data, including images and examination results.

\section{Results}

Clinical findings. Patient 1 had no known familial history of ocular disease. Refractive error was +4.50 diopter sphere (DS) in both eyes, with best corrected visual acuity (BVCA) at 1.0 in the right eye and 0.1 in the left eye. The cornea and the lens were transparent. Fundus examination revealed that the right eye had prominent yellow-white sub-retinal scarring with 
Table I. Summary of the primers and products length used for the amplification of the exons of bestrophin-1.

\begin{tabular}{cllrc}
\hline Exon & \multicolumn{1}{c}{ Forward (5'-3') } & \multicolumn{1}{c}{ Reverse $\left(5^{\prime}-3^{\prime}\right)$} & $\begin{array}{r}\text { Product } \\
\text { size }(\mathrm{bp})\end{array}$ & $\begin{array}{c}\text { Annealing } \\
\text { temperature }\left({ }^{\circ} \mathrm{C}\right)\end{array}$ \\
\hline 2 & AGTCTCAGCCATCTCCTCGC & TGGCCTGTCTGGAGCCTG & 212 & 61 \\
3 & GGGACAGTCTCAGCC ATCTC & CAGCTCCTCGTGATCCTCC & 238 & 58 \\
4 & AGAAAGCTGGAGGAGCCG & GCGGCAGCCCTGTCTGTAC & 1408 & 59 \\
5 & GGGGCAGGTGGTGTTCAGA & GGCAGCCTCACCAGCCTAG & 150 & 59 \\
6 & GGGCAGGTGGTGTTAGA & CCTTGGTCCTTCTAGCCTCAG & 181 & 59 \\
7 & CATCCTGATTTCAGGGTTCC & CTCTGGCCATGCCTCCAG & 257 & 59 \\
8 & AGCTGAGGTTTAAAGGGGGA & TCTCTTTGGGTCCACTTTGG & 215 & 59 \\
9 & ACATACAAGGTCCTGCCTGG & GCATTAACTAGTGCTATTCTAAGTTCC & 298 & 59 \\
$10 \mathrm{~A}$ & GGTGTTGGTCCTTGTCCAC & CTCTGGCATATCCGTCAGGT & 591 & 59 \\
$10 \mathrm{~B}$ & CTTCAAGTCTGCCCCACTGT & TAGGCTCAGAGCAAGGGAAG & 457 & 59 \\
11 & CATTTTGGTATTTGAAATGAAGG & CCATTTGATTCAGGCTGTTG & 216 & 59 \\
\hline
\end{tabular}

Bp, base pairs.
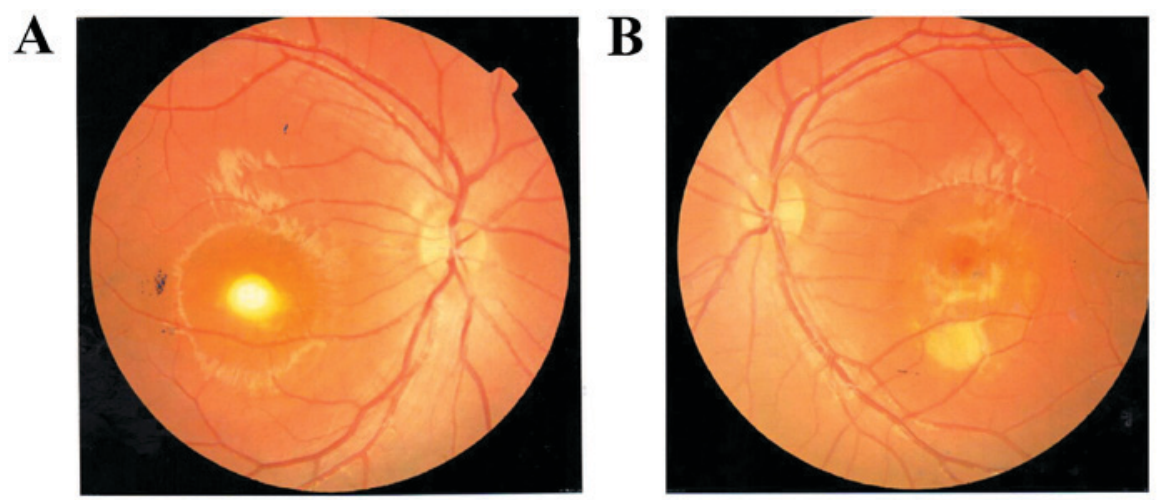

C
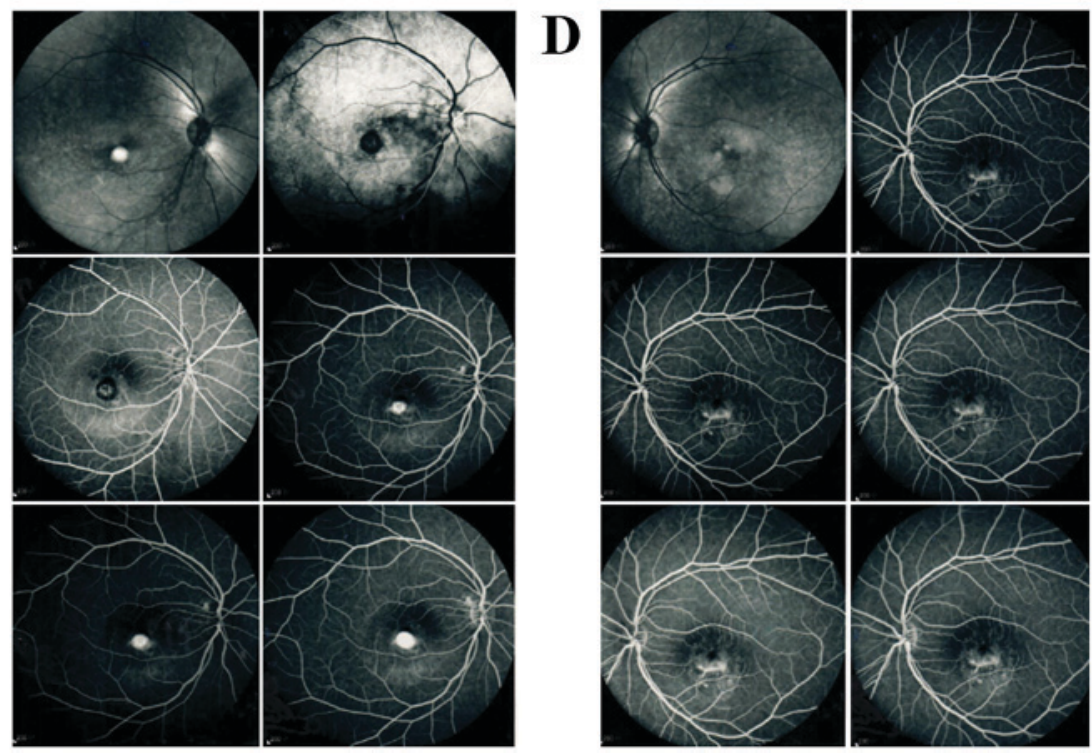

Figure 1. Fundus examination and FFA of Patient 1. (A and B) Fundus examination revealed the right eye had prominent yellow-white sub-retinal scarring with pigmented borders, surrounded by a serous retinal detachment. The left eye had fragmented vitelliform lesions. (C and D) FFA identified slight hyperfluorescence with mild leakage in the fovea of the macula in both eyes. FFA, fundus fluorescein angiography. (A and C) right eye; (B and D) left eye.

pigmented borders, surrounded by a serous retinal detachment. The left eye exhibited fragmented vitelliform lesions (Fig. 1A and B). FFA revealed a mild hyperfluorescence with moderate leakage in the fovea of the macula in both eyes (Fig. 1C and D). OCT scans revealed that the foveal region in both eyes was abnormally thick, due to neuroretinal 

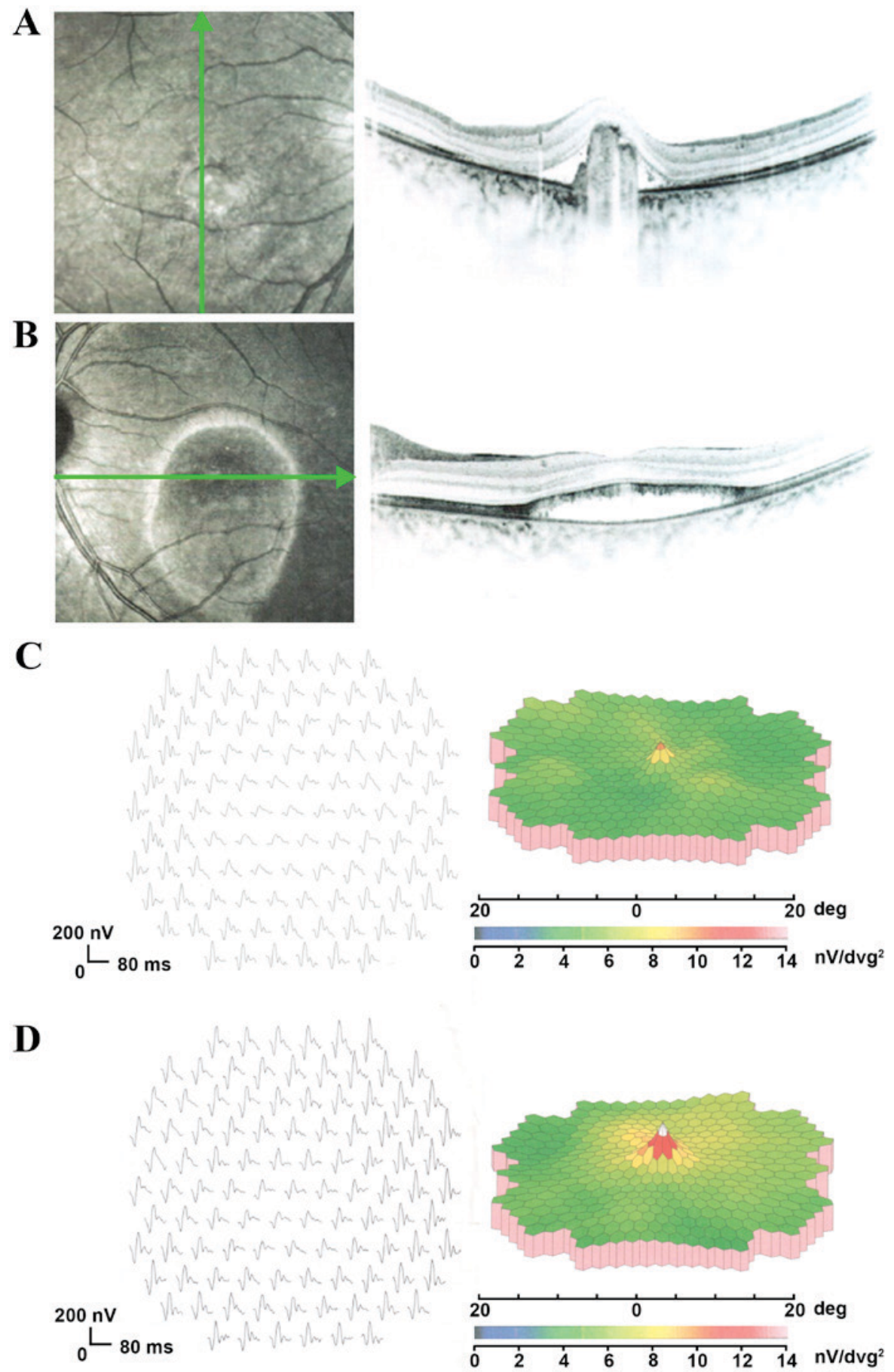

Figure 2. OCT scans and mfERG of Patient 1. (A and B) OCT scans revealed that the foveal region of the both eyes were abnormally thick due to neuroretinal detachment from the retinal pigment epithelium. (C and D) mfERGs (response of the posterior fundus) identified a mild decrease in the amplitude of the foveal response for each eye, while the peripheral mfERG amplitudes were within normal limits. OCT, optical coherence tomography; mfERG, multifocal electroretinography. (A and C) right eye; (B and D) left eye.

detachment from the RPE. In the right eye, the neuroretinal detachment was likely triggered by the abnormal accumulation of hyperreflective materials beneath the retina (Fig. 2A), whereas in the left eye, it was likely due to the accumulation of sub-retinal fluid (Fig. 2B). mfERGs (response of the posterior fundus) revealed a mild decrease in the amplitude of the foveal response in both eyes, although the peripheral mfERG amplitudes were within normal limits (Fig. 2C and D).

Patient 2 had myopia and their decline in vision had occurred over the last 3 years. Refractive error was -10.0 DS in the right eye and -9.0 DS in the left eye. The BVCA was 0.4 in the right eye and counting fingers at $50 \mathrm{~cm}$ away from the left eye. The cornea and the lens were transparent. Fundus examination revealed atrophic lesions in both eyes (Fig. 3A and B). FFA revealed significant early hyperfluorescence that had increased intensity at the late stage of the angiographic sequence, with mild leakage in the right eye (Fig. 3C). The macular lesions exhibited a dystrophic pattern in the left eye (Fig. 3D). OCT revealed that the foveal regions in both eyes were abnormally thin due to atrophy of the retina and RPE 

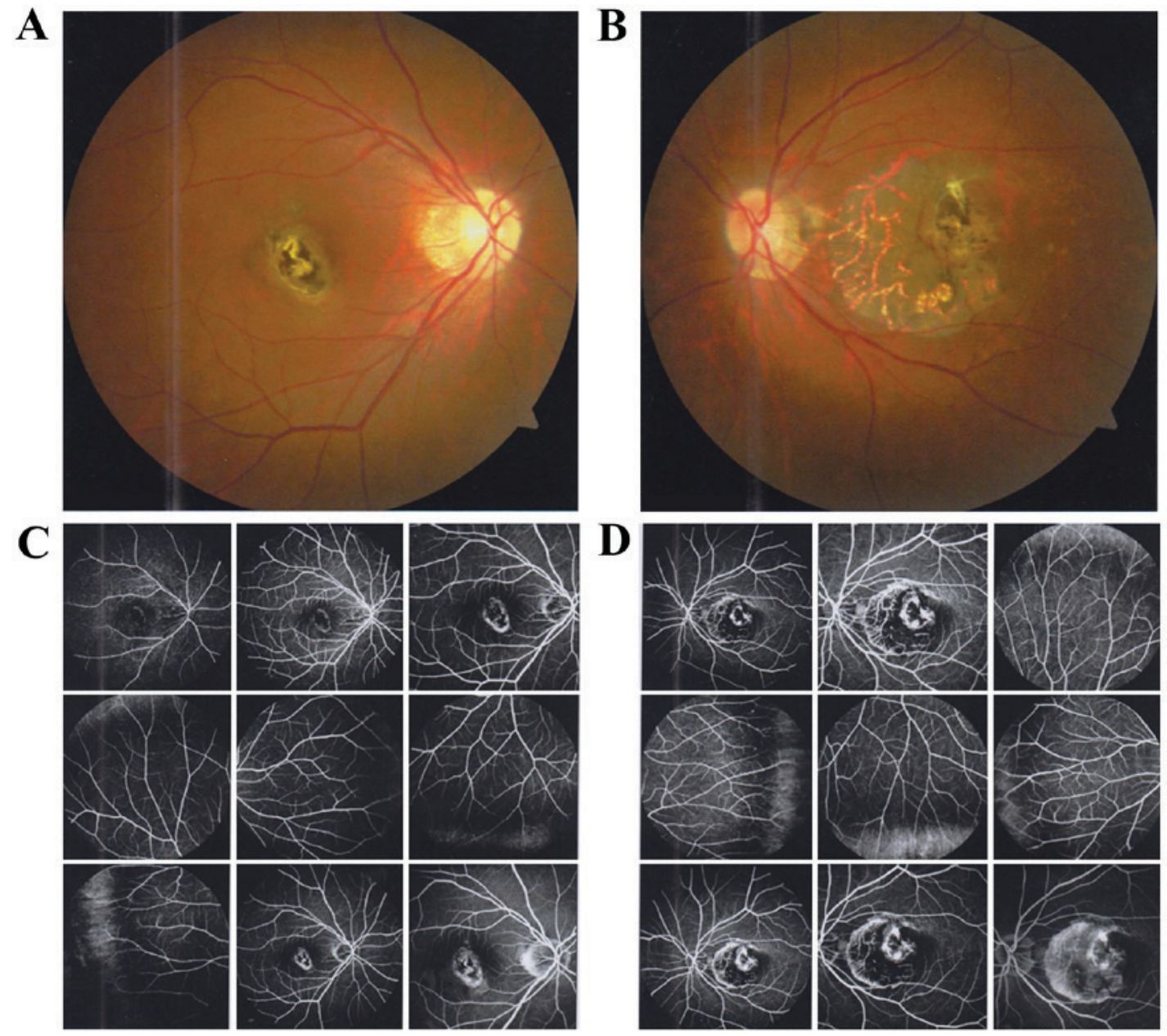

D

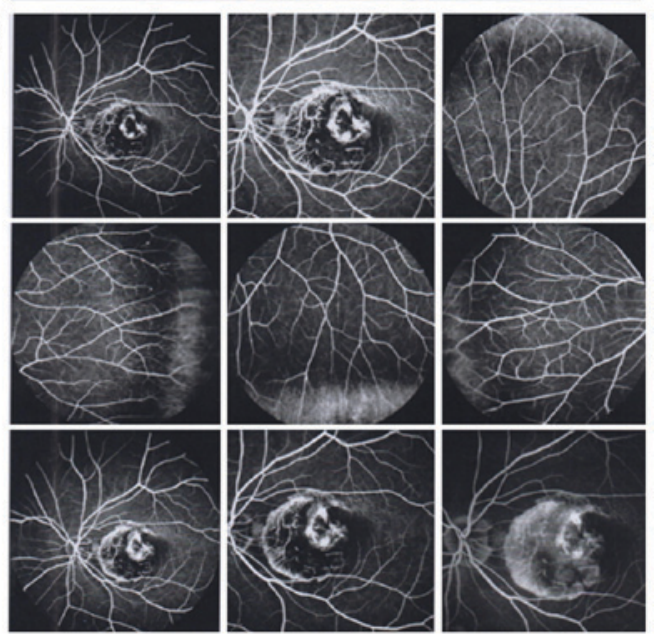

Figure 3. Fundus examination and FFA of Patient 2. (A and B) Fundus examination identified atrophic lesions in both eyes. (C and D) FFA revealed significant early hyperfluorescence with increased intensity at the late stage of the angiographic sequence. FFA, fundus fluorescein angiography. (A and C) right eye; (B and D) left eye.

(Fig. 4A and B). Similar to Patient 1, mfERGs of Patient 2 revealed a significant decrease in the amplitude of the foveal response in both eyes, although most of the peripheral mfERG amplitudes were within normal limits (Fig. 4C and D).

Mutation screening and bioinformatics analysis. A heterozygous mutation c.903T $>$ G (p.D301E) in exon 8 of the BESTl gene was identified in Patient 1, but not in the unaffected family members or the normal controls in the same population. A c.1608C $>$ T (p.T536T) single nucleotide polymorphism (SNP) in exon 10 of the BEST1 gene was identified in Patient 2 (Fig. 5A). Multiple sequence alignment performed using the HomoloGene database indicated that the residues at position 301 and 536 of bestrophin-1 are highly conserved (Fig. 5B). PolyPhen and SIFT predicted that the D301E amino acid substitution in bestrophin-1 was potentially damaging (Fig. 5C). SIFT predicted that the T536T substitution is tolerated.

\section{Discussion}

BVMD is a rare retinal disease with highly variable phenotypic expression (4). In the early stages of BVMD, patients are generally asymptomatic. As the disease progresses, slow and progressive vision loss occurs. In this study, both patients presented typical BVMD macular phenotypes. However, several extramacular lesions were also present. Hyperopia greater than +3.00 diopters is a recognized feature of juvenile-onset BVMD $(31,40)$. In this study, Patient 1 exhibited hyperopia, whereas Patient 2 exhibited myopia. Other anterior segment abnormalities, including shallow anterior chambers, closed or narrow anterior chamber angles and angle-closure glaucoma may also be present in BVMD (41).

Adult-onset BVMD was first described by Gass in 1974 (42). Compared with juvenile-onset BVMD, adult-onset BVMD is less well-characterized (43). It usually occurs between 30-50 years of age, and is often associated with bilateral macular dystrophy and sub-retinal oval or round yellowish deposition in the macula (44). A definite diagnosis of adult-onset BVMD is often difficult. It is considered as a subtype of pattern dystrophy (PD) (43). Atypical presentations including multifocal vitelliform lesions and the occurrence of choroidal neovascularization (CNV) in various disease stages may confound diagnosis $(11,12,45,46)$. Adult-onset BVMD can also be accompanied by branched retinal vein occlusion (47). Patient 2 was diagnosed with CNV in 2014, and her visual acuity improved following treatment with ranibizumab injection. Although this patient presented 


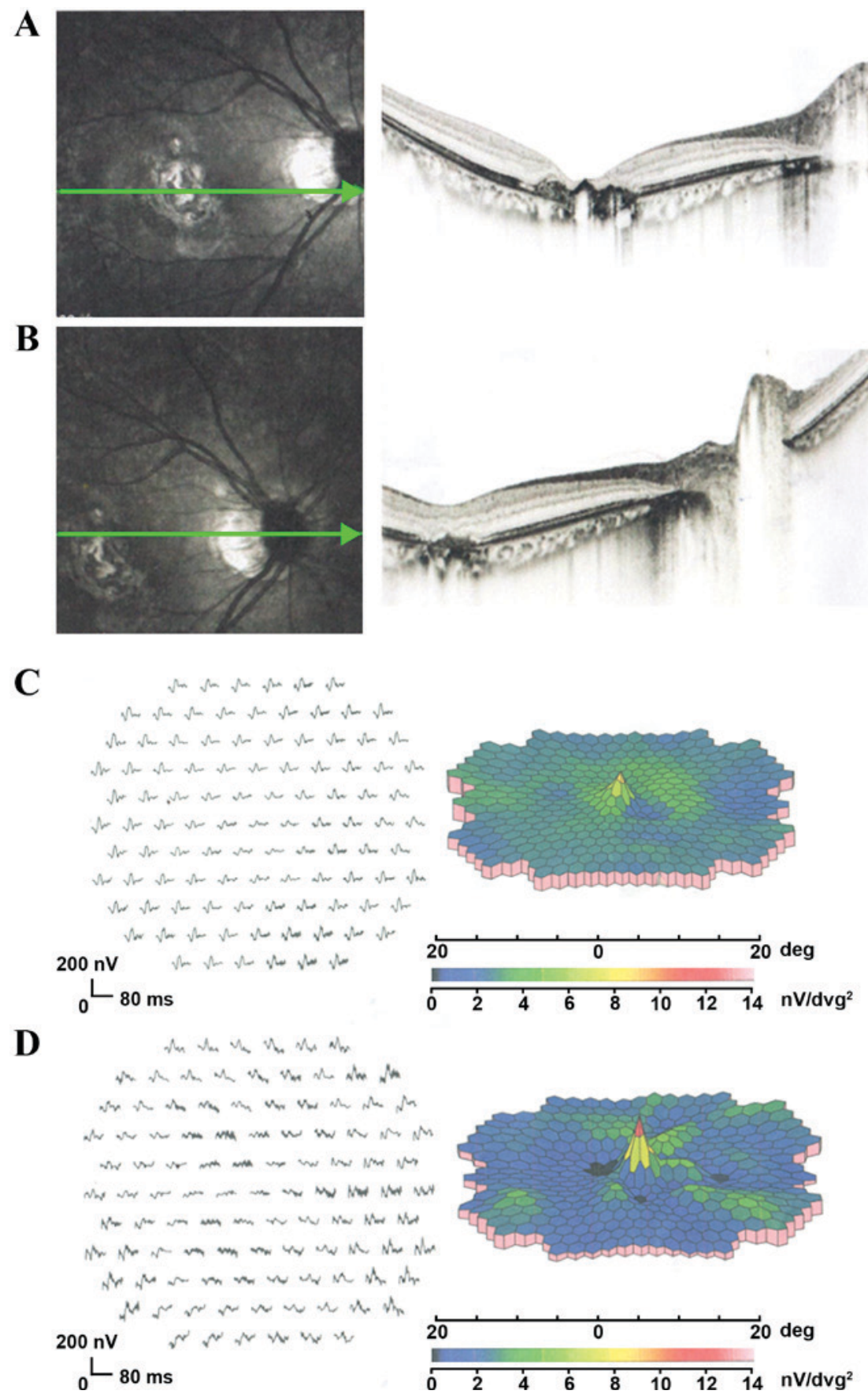

Figure 4. OCT scans and mfERG of Patient 2. (A and B) OCT revealed that the foveal region of both eyes was abnormally thin due to atrophy of retina and the RPE. (C and D) mfERGs identified a significant decrease in the amplitude of the foveal response for each eye, while most of the peripheral mfERG amplitudes were within normal limits. OCT, optical coherence tomography; mfERG, multifocal electroretinography. (A and C) right eye; (B and D) left eye.

typical PD, genetic examination was helpful to confirm the diagnosis following the exclusion of other causes of macular atrophy.

BEST1 gene mutations and polymorphisms have been reported in diverse ethnic groups $(15,48-50)$. In this study, one recurrent mutation, c.903T>G (p.D301E) in exon 8, and one SNP, c.1608C >T (p.T536T), in exon 10 were reported. Caldwell et al (48) and Allikmets et al (49) also identified several other SNPs in the BEST1 gene of patients with BVMD, including Y29Y, L37L, A10A, I73I, R218R, P341P, T470T and S519S. As the cellular function of bestrophin-1 protein is not fully elucidated, it is challenging to predict which mutations are pathological and which are benign. A recent study using patient-derived RPE cells demonstrated that a V235A mutation can lead to the mislocalization of bestrophin-1 from the basolateral membrane to the apical membrane and potentially disrupt the polarized conductance (51). Furthermore, the R218H, L234P and A243T mutations result in defective calcium-activated chloride ion export (52). The D301E mutation identified in this study is located at the $\mathrm{C}$ terminus of bestrophin-1. The amino acids between position 292-312 are frequently mutated (Fig. 6). Mutations may disrupt the interaction between the $\mathrm{N}$ and $\mathrm{C}$ termini, which subsequently alters chloride channel function (53). 
A

Normal

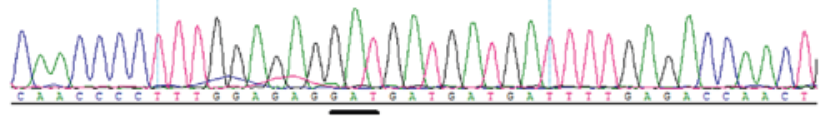
| c.903T>G (p.D301E)

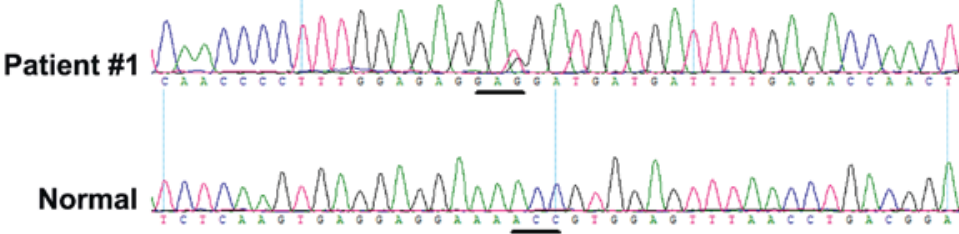

Patient \#2

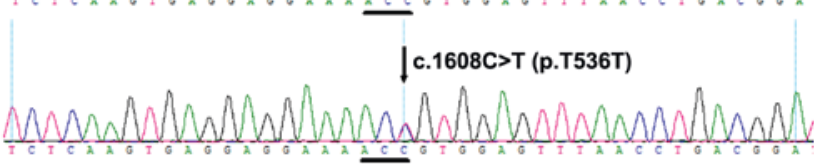

B

Whole sequence identity

\begin{tabular}{|c|c|c|c|c|}
\hline & DNA & Protein & 301 & 536 \\
\hline H.sapiens & $100 \%$ & $100 \%$ & AEQLINPFGEDDDDFETNWIV & QVRRKTVEFNLTDMPE \\
\hline C.lupus & $86 \%$ & $81 \%$ & AEQLINPFGEDDDDFETNWIV & NPESGHVKRKTVEFNLTDMSG \\
\hline taurus & $85 \%$ & $81 \%$ & AEQLINPFGEDDDDFETNWIV & HPEVIHMRRKTVEFNLMDMSE \\
\hline musculus & $77 \%$ & $70 \%$ & AEQLINPF & E \\
\hline norvegicus & $77 \%$ & $70 \%$ & AEQLINPFGEDDDDFETNWI I & YQEACH \\
\hline
\end{tabular}

C

D301E

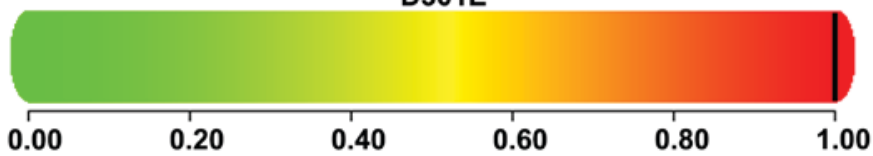

This mutation is predicted to be PROBABLY DAMAGING with a score of 1.000

Figure 5. Identification of one recurrent mutation in Patient 1 and one SNP in Patient 2. (A) A heterozygous mutation c.903T $>$ G (p.D301E) in exon 8 of the BEST1 gene was identified in Patient 1, but not in unaffected family members or the normal controls in the same population. A c.1608C >T (p.T536T) SNP in exon 10 of the BEST1 gene was identified in Patient 2. (B) Multiple-sequence alignment of the BEST1 genes and besotrophin-1 proteins from different species. The red triangles indicate the position of the mutation and the SNP. The amino acid residues at these two positions are conserved across species. (C) Polyphen predicted that the amino acid substitution D301E in the protein bestrophin-1 is probably damaging. SNP, single nucleotide polymorphism, BEST1, bestrophin-1 gene.

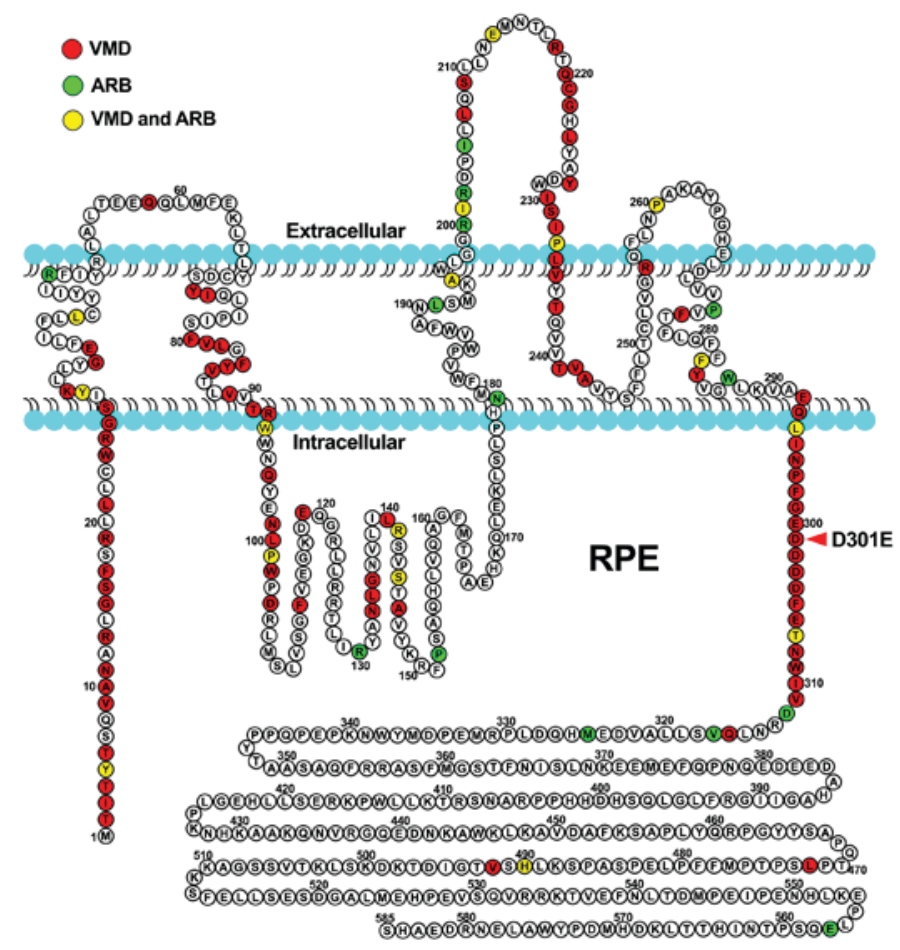

Figure 6. Schematic diagram of the location of identified bestrophin-1 protein mutations. The topology of human bestrophin-1 was drawn according to the model by Tsunenari et al $(54,55)$. Red circles indicate mutated amino acids identified in VMD. Green circles indicate mutated amino acids identified in ARB. Yellow circles indicate mutated amino acids identified in both VMD and ARB. Data acquired from UniProt (www.uniprot.org) and http://www.retina-international.org/mutations-best1-gene. VMD, vitelliform macular dystrophy; ARB, autosomal recessive bestrophinopathy; RPE, retinal pigmented epithelium. 
In conclusion, two recurrent genetic variations of BEST1 in two Chinese patients with either juvenile-onset BVMD or adult-onset BVMD were identified in this study. These findings expand the mutation spectrum of BEST1 and may aid in genetic counseling as well as prenatal diagnoses of patients with BVMD. Additionally, characterization of these genetic variations in BVMD provides a basis for future investigation of the underlying disease pathogenesis and the development of therapeutic interventions.

\section{Acknowledgements}

The present study was supported by the National Natural Science Foundation of China (grant nos. 81500709, 81570862 and 81670872) and the State Scholarship Fund from the China Scholarship Council.

\section{References}

1. Johnson AA, Guziewicz KE, Lee CJ, Kalathur RC, Pulido JS, Marmorstein LY and Marmorstein AD: Bestrophin 1 and retinal disease. Prog Retin Eye Res 58: 45-69, 2017.

2. Xiao Q, Hartzell HC and Yu K: Bestrophins and retinopathies. Pflugers Arch 460: 559-569, 2010.

3. Qian CX, Charran D, Strong CR, Steffens TJ, Jayasundera T and Heckenlively JR: Optical coherence tomography examination of the retinal pigment epithelium in best vitelliform macular dystrophy. Ophthalmology 124: 456-463, 2017.

4. Guziewicz KE, Sinha D, Gómez NM, Zorych K, Dutrow EV, Dhingra A, Mullins RF, Stone EM, Gamm DM, Boesze-Battaglia K and Aguirre GD: Bestrophinopathy: An RPE-photoreceptor interface disease. Prog Retin Eye Res 58: 70-88, 2017.

5. Arora R, Khan K, Kasilian ML, Strauss RW, Holder GE, Robson AG, Thompson DA, Moore AT and Michaelides M: Unilateral BEST1-associated retinopathy. Am J Ophthalmol 169: 24-32, 2016.

6. Subash M, Rotsos T, Wright GA, Devery S, Holder GE, Robson AG, Pal B, Tufail A, Webster AR, Moore AT and Michaelides M: Unilateral vitelliform maculopathy: A comprehensive phenotype study with molecular screening of BEST1 and PRPH2. Br J Ophthalmol 96: 719-722, 2012.

7. Liu J, Xuan Y, Zhang Y, Liu W and Xu G: Bilateral macular holes and a new onset vitelliform lesion in Best disease. Ophthalmic Genet 38: 79-82, 2017.

8. Elkhoyaali A, Chatoui S, Bercheq N, Elouatassi N, Zerrouk R, Elasri F, Reda K and Oubaaz A: Choroidal neovascularization complicating Best's vitelliform macular dystrophy in a child. J Fr Ophtalmol 39: 69-73, 2016.

9. Peiretti E, Caminiti G, Forma G, Carboni G, Dhaenens CM, Querques L, Souied E and Querques G: A Novel p. Asp304Gly Mutation In Best1 gene associated with atypical best vitelliform macular dystrophy phenotype and high intrafamilial variability. Retina 36: 1733-1740, 2016.

10. Chacon-Camacho OF, Camarillo-Blancarte L and Zenteno JC: OCT findings in young asymptomatic subjects carrying familial BEST1 gene mutations. Ophthalmic Genet 32: 24-30, 2011.

11. Stanca HT and Nicolaescu M: Adult onset foveomacular vitelliform dystrophy. Oftalmologia 55: 82-86, 2011.

12. Brecher R and Bird AC: Adult vitelliform macular dystrophy. Eye (Lond) 4: 210-215, 1990.

13. Liu J, Zhang Y, Xuan Y, Liu W and Wang M: Novel BEST1 Mutations and special clinical features of best vitelliform macular dystrophy. Ophthalmic Res 56: 178-185, 2016.

14. Lin Y, Li T, Gao H, Lian Y, Chen C, Zhu Y, Li Y, Liu B, Zhou W, Jiang $\mathrm{H}$, et al: Bestrophin 1 gene analysis and associated clinical findings in a Chinese patient with Best vitelliform macular dystrophy. Mol Med Rep 16: 4751-4755, 2017.

15. Krämer F, White K, Pauleikhoff D, Gehrig A, Passmore L, Rivera A, Rudolph G, Kellner U, Andrassi M, Lorenz B, et al: Mutations in the VMD2 gene are associated with juvenile-onset vitelliform macular dystrophy (Best disease) and adult vitelliform macular dystrophy but not age-related macular degeneration. Eur J Hum Genet 8: 286-292, 2000.
16. Glavač D, Jarc-Vidmar M, Vrabec K, Ravnik-Glavač M, Fakin A and Hawlina M: Clinical and genetic heterogeneity in Slovenian patients with BEST disease. Acta Ophthalmol 94: e786-e794, 2016.

17. Matson ME, Ly SV and Monarrez JL: Novel Mutation in BEST1 Associated with Atypical Best Vitelliform Dystrophy. Optom Vis Sci 92: e180-e189, 2015.

18. Pasquay C, Wang LF, Lorenz B and Preising MN: Bestrophin 1-phenotypes and functional aspects in bestrophinopathies. Ophthalmic Genet 36: 193-212, 2015.

19. Seddon JM, Afshari MA, Sharma S, Bernstein PS, Chong S, Hutchinson A, Petrukhin K and Allikmets R: Assessment of mutations in the Best macular dystrophy (VMD2) gene in patients with adult-onset foveomacular vitelliform dystrophy, age-related maculopathy, and bull's-eye maculopathy. Ophthalmology 108: 2060-2067, 2001.

20. Marmorstein AD, Kinnick TR, Stanton JB, Johnson AA, Lynch RM and Marmorstein LY: Bestrophin-1 influences transepithelial electrical properties and $\mathrm{Ca} 2+$ signaling in human retinal pigment epithelium. Mol Vis 21: 347-359, 2015.

21. Tan X, Zhu Y, Chen C, Chen X, Qin Y, Qu B, Luo L, Lin H, Wu M, Chen W and Liu Y: Sprouty2 Suppresses Epithelial-Mesenchymal transition of human lens epithelial cells through Blockade of Smad2 and ERK1/2 pathways. PLoS One 11: e0159275, 2016.

22. Zhang Y, Morgan R, Chen C, Cai Y, Clark E, Khan WN, Shin SU, Cho HM, Al Bayati A, Pimentel A and Rosenblatt JD: Mammary-tumor-educated B cells acquire LAP/TGF- $\beta$ and PD-L1 expression and suppress anti-tumor immune responses. Int Immunol 28: 423-433, 2016.

23. Qin Y, Zhu Y, Luo F, Chen C, Chen X and Wu M: Killing two birds with one stone: Dual blockade of integrin and FGF signaling through targeting syndecan- 4 in postoperative capsular opacification. Cell Death Dis 8: e2920, 2017.

24. Tan X, Chen C, Zhu Y, Deng J, Qiu X, Huang S, Shang F, Cheng B and Liu Y: Proteotoxic stress desensitizes TGF-beta signaling through receptor downregulation in retinal pigment epithelial cells. Curr Mol Med: Jun 19, 2017 (Epub ahead of print).

25. Tan X, Zhan J, Zhu Y, Cao J, Wang L, Liu S, Wang Y, Liu Z, Qin Y, Wu M, et al: Improvement of uveal and capsular biocompatibility of hydrophobic acrylic intraocular lens by surface grafting with 2-Methacryloyloxyethyl phosphorylcholine-methacrylic acid copolymer. Sci Rep 7: 40462, 2017.

26. Aldehni F, Spitzner M, Martins JR, Barro-Soria R, Schreiber R and Kunzelmann K: Bestrophin 1 promotes epithelial-to-mesenchymal transition of renal collecting duct cells. J Am Soc Nephrol 20: 1556-1564, 2009.

27. Oh SJ and Lee CJ: Distribution and function of the bestrophin-1 (Best1) Channel in the Brain. Exp Neurobiol 26: 113-121, 2017.

28. Xi L, Liu Y, Zhao F, Chen C and Cheng B: Analysis of glistenings in hydrophobic acrylic intraocular lenses on visual performance. Int J Ophthalmol 7: 446-451, 2014.

29. Lin Y, Gao H, Ai S, Eswarakumar JVP, Chen C, Zhu Y, Li T, Liu B, Liu X, Luo L, et al: C278F mutation in FGFR2 gene causes two different types of syndromic craniosynostosis in two Chinese patients. Mol Med Rep 16: 5333-5337, 2017.

30. Lin Y, Gao H, Ai S, Eswarakumar JV, Zhu Y, Chen C, Li T, Liu B, Jiang H, Liu Y, et al: FGFR2 mutations and associated clinical observations in two Chinese patients with Crouzon syndrome. Mol Med Rep: Aug 29, 2017 (Epub ahead of print).

31. Lin Y, Gao H, Liu Y, Liang X, Liu X, Wang Z, Zhang W, Chen J, Lin Z, Huang $X$ and Liu Y: Two novel mutations in the bestrophin-1 gene and associated clinical observations in patients with best vitelliform macular dystrophy. Mol Med Rep 12: 2584-2588, 2015.

32. Lin Y, Ai S, Chen C, Liu X, Luo L, Ye S, Liang X, Zhu Y, Yang H and Liu Y: Ala344Pro mutation in the FGFR2 gene and related clinical findings in one Chinese family with Crouzon syndrome. Mol Vis 18: 1278-1282, 2012.

33. Lin Y, Liang X, Ai S, Chen C, Liu X, Luo L, Ye S, Li B, Liu Y and Yang H: FGFR2 molecular analysis and related clinical findings in one Chinese family with Crouzon syndrome. Mol Vis 18: 449-454, 2012.

34. Lin Y, Liu X, Yu S, Luo L, Liang X, Wang Z, Chen C, Zhu Y, Ye S, Yan $\mathrm{H}$ and Liu Y: PAX6 analysis of two sporadic patients from southern China with classic aniridia. Mol Vis 18: 2190-2194, 2012.

35. Adzhubei IA, Schmidt S, Peshkin L, Ramensky VE, Gerasimova A, Bork P, Kondrashov AS and Sunyaev SR: A method and server for predicting damaging missense mutations. Nat Methods 7: 248-249, 2010. 
36. Salvo J, Lyubasyuk V, Xu M, Wang H, Wang F, Nguyen D, Wang K, Luo H, Wen C, Shi C, et al: Next-generation sequencing and novel variant determination in a cohort of 92 familial exudative vitreoretinopathy Patients. Invest Ophthalmol Vis Sci 56: 1937-1946, 2015.

37. Nikopoulos K, Gilissen C, Hoischen A, van Nouhuys CE, Boonstra FN, Blokland EA, Arts P, Wieskamp N, Strom TM, Ayuso C et al: Next-generation sequencing of a $40 \mathrm{Mb}$ linkage interval reveals TSPAN12 mutations in patients with familial exudative vitreoretinopathy. Am J Hum Genet 86: 240-247, 2010.

38. Kumar P, Henikoff S and Ng PC: Predicting the effects of coding non-synonymous variants on protein function using the SIFT algorithm. Nat Protoc 4: 1073-1081, 2009.

39. Avila-Fernandez A, Perez-Carro R, Corton M, Lopez-Molina MI, Campello L, Garanto A, Fernandez-Sanchez L, Duijkers L, Lopez-Martinez MA, Riveiro-Alvarez R, et al: Whole-exome sequencing reveals ZNF408 as a new gene associated with autosomal recessive retinitis pigmentosa with vitreal alterations. Hum Mol Genet 24: 4037-4048, 2015.

40. Wivestad Jansson R, Berland S, Bredrup C, Austeng D, Andréasson S and Wittström E: Biallelic mutations in the BEST1 gene: Additional families with autosomal recessive bestrophinopathy. Ophthalmic Genet 37: 183-193, 2016.

41. Wittström E, Ponjavic V, Bondeson ML and Andréasson S: Anterior segment abnormalities and angle-closure glaucoma in a family with a mutation in the BEST1 gene and Best vitelliform macular dystrophy. Ophthalmic Genet 32: 217-227, 2011.

42. Gass JD: A clinicopathologic study of a peculiar foveomacular dystrophy. Trans Am Ophthalmol Soc 72: 139-156, 1974.

43. Chowers I, Tiosano L, Audo I, Grunin M and Boon CJ: Adult-onset foveomacular vitelliform dystrophy: A fresh perspective. Prog Retin Eye Res 47: 64-85, 2015.

44. Tiosano L, Grunin M, Hagbi-Levi S, Banin E, Averbukh E and Chowers I: Characterising the phenotype and progression of sporadic adult-onset foveomacular vitelliform dystrophy. Br J Ophthalmol 100: 1476-1481, 2016.

45. Rishi E, Rishi $P$ and Mahajan S: Intravitreal bevacizumab for choroidal neovascular membrane associated with Best's vitelliform dystrophy. Indian J Ophthalmol 58: 160-162, 2010.

46. Mimoun G, Caillaux V, Querques G, Rothschild PR, Puche N and Souied EH: Ranibizumab for choroidal neovascularization associated with adult-onset foveomacular vitelliform dystrophy: One-year results. Retina 33: 513-521, 2013.

47. Lee CS, Jun I, Choi SI, Lee JH, Lee MG, Lee SC and Kim EK: A Novel BEST1 mutation in autosomal recessive bestrophinopathy. Invest Ophthalmol Vis Sci 56: 8141-8150, 2015.
48. Caldwell GM, Kakuk LE, Griesinger IB, Simpson SA, Nowak NJ, Small KW, Maumenee IH, Rosenfeld PJ, Sieving PA, Shows TB and Ayyagari R: Bestrophin gene mutations in patients with Best vitelliform macular dystrophy. Genomics 58: 98-101, 1999.

49. Allikmets R, Seddon JM, Bernstein PS, Hutchinson A, Atkinson A, Sharma S, Gerrard B, Li W, Metzker ML, Wadelius C, et al: Evaluation of the best disease gene in patients with age-related macular degeneration and other maculopathies. Hum Genet 104: 449-453, 1999.

50. Li T, Lin Y, Gao H, Chen C, Zhu Y, Liu B, Lian Y, Li Y, Zhou W, Jiang $\mathrm{H}$, et al: Two heterozygous mutations identified in one Chinese patient with bilateral macular coloboma. Mol Med Rep 16: 2505-2510, 2017.

51. Carter DA, Smart MJ, Letton WV, Ramsden CM, Nommiste B, Chen LL, Fynes K, Muthiah MN, Goh P, Lane A, et al: Mislocalisation of BEST1 in iPSC-derived retinal pigment epithelial cells from a family with autosomal dominant vitreoretinochoroidopathy (ADVIRC). Sci Rep 6: 33792, 2016.

52. Moshfegh Y, Velez G, Li Y, Bassuk AG, Mahajan VB and Tsang SH: BESTROPHIN1 mutations cause defective chloride conductance in patient stem cell-derived RPE. Hum Mol Genet 25: 2672-2680, 2016.

53. Qu Z, Cheng W, Cui Y, Cui Y and Zheng J: Human disease-causing mutations disrupt an NC-terminal interaction and channel function of bestrophin 1. J Biol Chem 284: 16473-16481, 2009.

54. Hartzell HC, Qu Z, Yu K, Xiao Q and Chien LT: Molecular physiology of bestrophins: Multifunctional membrane proteins linked to best disease and other retinopathies. Physiol Rev 88: 639-672, 2008.

55. Tsunenari T, Sun H, Williams J, Cahill H, Smallwood P, Yau KW and Nathans J: Structure-function analysis of the bestrophin family of anion channels. J Biol Chem 278: 41114-41125, 2003.

This work is licensed under a Creative Commons Attribution-NonCommercial-NoDerivatives 4.0 International (CC BY-NC-ND 4.0) License. 